\title{
Touch Key Design for Target Selection on a Mobile Phone
}

\author{
Yong S. Park ${ }^{1}$, Sung H. Han ${ }^{2}$, Jaehyun Park ${ }^{3}$, Youngseok Cho ${ }^{4}$ \\ Department of Industrial and Management Engineering \\ Pohang University of Science and Technology (POSTECH) \\ San 31 Hyoja, Pohang, South Korea 790-784 \\ $+82-54-279-2862^{1,3,4},+82-54-279-2203^{2}$ \\ $\left\{\right.$ drastle ${ }^{1}, \operatorname{shan}^{2}$, parkdog $3^{3}$, kilys $\left.^{4}\right\}$ @postech.ac.kr
}

\begin{abstract}
Mobile phones with a touch screen replacing traditional keypads have been introduced to the market. Few studies, however, have been conducted on the touch interface design for a mobile phone. This study investigated the effects of touch key sizes and locations on the one-handed thumb input that is popular in mobile phone interactions. Three different touch key sizes (i.e. square shape with $4 \mathrm{~mm}, 7 \mathrm{~mm}$, and $10 \mathrm{~mm}$ wide) and twenty five locations were examined in an experiment. The results provided two groups of touch key locations (an appropriate and an inappropriate region) with respect to three usability measures including success rate, number of errors, and pressing convenience. In addition, a hits distributions based algorithm was applied to target selection tasks, which statistically improved the performance. The results of this study could be used to design touch keys so as to enhance the usability of mobile phones with a touch screen.
\end{abstract}

\section{Categories and Subject Descriptors}

H.5.2 [Information Interfaces and Presentation]: User Interfaces - Ergonomics, Input devices and strategies.

\section{General Terms}

Algorithms, Performance, Design, Experimentation, Human Factors

\section{Keywords}

One-handed thumb input, mobile phones, touch screen, hits distribution based algorithm, usability

\section{INTRODUCTION}

Touch screens are widely used for a variety of mobile devices because they are highly intuitive and require little space to implement [1]. Moreover, touch interfaces are easy to adjust the design parameters, such as key size, spacing between keys and location on the screen. Recently, mobile phones with a touch screen replacing traditional keypads, e.g. Apple ${ }^{\mathrm{TM}}$ iPhone, are coming into the spotlight.

Users tend to use only one hand when they use a mobile device

Permission to make digital or hard copies of all or part of this work for personal or classroom use is granted without fee provided that copies are not made or distributed for profit or commercial advantage and that copies bear this notice and the full citation on the first page. To copy otherwise, or republish, to post on servers or to redistribute to lists, requires prior specific permission and/or a fee.

MobileHCI 2008, September 2-5, 2008, Amsterdam, the Netherlands.

Copyright $\odot 2008$ ACM 978-1-59593-952-4/08/09...\$5.00.
[4]. In other words, they hold a mobile phone with one hand and interact with it using a thumb. In addition, they would use both hands only when the user interface makes one hand interaction impossible or difficult.

It is difficult to find studies investigating critical design factors such as touch key size, touch key location, and touch recognition area on a mobile device, although one handed interaction on a mobile device is popular. A previous study investigated onehanded thumb input on a PDA [6]. This study divided a PDA screen into $3 \times 3$ areas and examined usability of each region. However, the results for the nine areas are not enough to be used to design a mobile phone interface. Note that, mobile phones in use often provide more than nine input elements simultaneously. For example, an Apple ${ }^{\mathrm{TM}}$ iPhone can provide more than three items in a row and in a column.

Input accuracy is critical to designing a mobile phone interface since people are using mobile phones frequently for a variety of purposes. Studies on improving input accuracy have been performed for many interfaces, e.g. virtual keyboarding [3] and a touch screen based keyboard [7].

This study aims to understand one-handed thumb input on a touch screen and to enhance usability of a mobile phone with a touch screen. To achieve the purposes, a human factors experiment is conducted to investigate the effects of touch key sizes and locations on usability of one-handed touch input. In addition, an algorithm based on hits distribution is applied in order to support user's input and to improve touch input accuracy.

\section{Experimental Methods}

\subsection{Subjects}

A total of thirty right-handed subjects participated in a human factors experiment. Their age ranged from 18 to 28 years old (mean $=23.1, \mathrm{SD}=2.5$ ). They had normal vision and no problem to freely move their right thumb. Twenty of them had not used a mobile device with a touch screen (e.g. personal digital assistants), while the others had experienced for 1.2 years on the average.

\subsection{Experimental design}

A within-subject design was used in the experiment, in which two within-subject variables (touch key size and touch key location) were included. The touch key size factor had three levels (square shape with $4 \mathrm{~mm}, 7 \mathrm{~mm}$, and $10 \mathrm{~mm}$ wide). A pilot test revealed the 
three touch key sizes represented small, medium and large touch key sizes, respectively.

The touch key location factor had 25 different levels. Each location was one of center points of 25 rectangular areas that had the same width and height (that is, one-fifth of a touch screen width and one-fifth of a touch screen height, respectively). Each area had its own ID (See Figure 3). In the experiment, the center point of a square touch key was positioned one of 25 touch key locations. That is, although touch keys had different sizes, the center points of them were located on the same position when they are located in the same touch key location. Figure 1 illustrated an example of experimental conditions, which had a touch key size of $10 \mathrm{~mm}$.

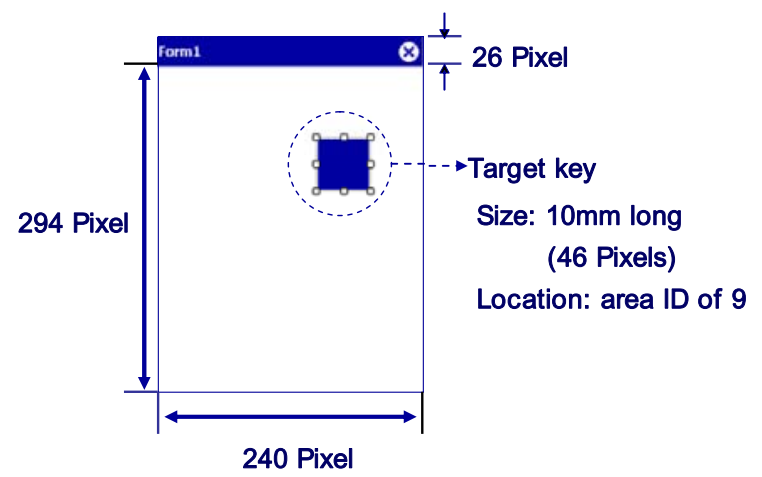

Figure 1. An example of experimental tasks

\subsection{Dependent measures}

Two types of dependent measures (pressing performance and a subjective satisfaction score) were collected in the study. The pressing performance measures included success rate, number of errors, and pressing deviation. The success rate was calculated based on the number of tasks correctly pressed at the first press. The pressing deviation was calculated by the difference between a center of a target and a centroid of a pressed area.

In order to obtain the subjective satisfaction score, each subject was asked to rate pressing convenience for each experimental condition using a nine point rating scale. The pressing convenience meant how easily the subjects could press a target.

\subsection{Apparatus}

A commercial PDA with a touch screen size of $240 \times 320$ pixels $\left(\mathrm{HP}^{\mathrm{TM}}\right.$ iPAQ rz1717) was used to implement an experimental prototype because there were a few mobile phones that equipped touch screens which could be easily manipulated in the experiment. It also had smaller body size than other mobile devices with touch screens (e.g. PMPs and other PDAs), which could provide a device size similar to real mobile phones

\subsection{Experimental tasks}

Each experimental task consisted of two states, a stand-by state and an input state. In the stand-by state, the experimental prototype was waiting for any user press on a touch screen. If a subject pressed any location on a touch screen in the stand-by state, the state changed to an input state after 0.3 second delay. That is, a blue touch key, a target key, was presented. In case that a subject pressed the blue key correctly, a 'beep' sound was provided. Then a stand-by state for the next task started. If a subject failed to press the target correctly, a pressed location for every press was recorded and no response was provided by an incorrect press. Subjects who failed to press the target correctly were asked to press the targets until they succeeded.

\subsection{Experimental procedure}

Each subject was given written instructions on the experimental objectives and procedures at the beginning of the experiment. Then he/she was asked to hold a PDA with his/her right hand and to practice pressing targets by the right thumb. In case that thumb movements by the right hand were interfered by the PDA body, the subject was allowed to put his/her left hand underneath the PDA in order to support easy and free thumb movements like real mobile phone use. Examples for the two methods to hold a PDA were presented in Figure 2.
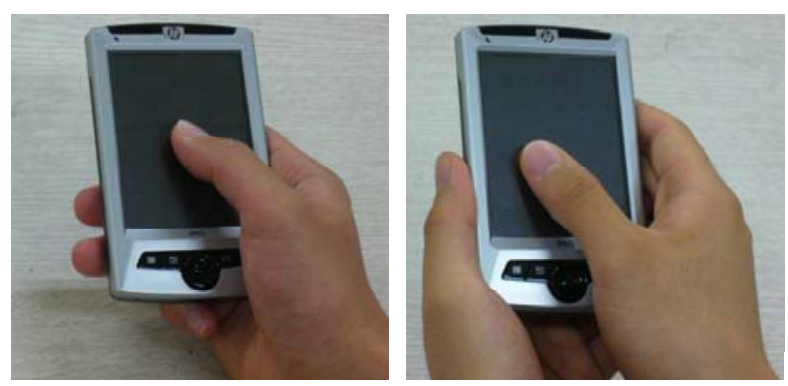

Figure 2. Two methods to hold a PDA. Left and Right figures showed one-hand and two-hands holding, respectively.

The main experiment consisted of three blocks. Each block consisted of 25 experimental conditions (i.e. 25 different touch key locations with the same touch key size). For each experimental condition, the two-state experimental task was repeated 10 times. That is, each subject carried out a total of 250 tasks in each block. After completing each block, the subjects rated pressing convenience for 25 touch key locations. The presentation order of three blocks was determined by the Latinsquare balancing technique.

\section{Results}

The ANOVA on ranks, one of non-parametric statistical techniques, was applied to two error related measures, the success rate and the number of errors, and the pressing convenience because of non-parametric characteristics of the data [2]. The results showed all measures were affected by the touch key size and the touch key location. Table 1 showed the ANOVA results including F-statistic and p-value. 
Table 1. Summary of the ANOVA on ranks results

\begin{tabular}{|c|c|c|}
\hline & Touch key size & Touch key location \\
\hline Success rate & $\begin{array}{c}\mathrm{F}(2,58)=437.1, \\
\mathrm{p}<0.01\end{array}$ & $\begin{array}{c}\mathrm{F}(24,696)=6.6, \\
\mathrm{p}<0.01\end{array}$ \\
\hline Number of errors & $\begin{array}{c}\mathrm{F}(2,58)=501.0, \\
\mathrm{p}<0.01\end{array}$ & $\begin{array}{c}\mathrm{F}(24,696)=6.5, \\
\mathrm{p}<0.01\end{array}$ \\
\hline $\begin{array}{c}\text { Pressing } \\
\text { Convenience }\end{array}$ & $\begin{array}{c}\mathrm{F}(2,58)=57.1, \\
\mathrm{p}<0.01\end{array}$ & $\begin{array}{c}\mathrm{F}(24,696)=75.34 \\
\mathrm{p}<0.001\end{array}$ \\
\hline
\end{tabular}

As post-hoc analyses, the Student Newman-Keuls (SNK) tests were conducted on significant main effects (i.e. the touch key size and the touch key location) at the 0.05 significance level. The results revealed, as expected, the number of errors decreased as the touch key size increased. In addition, it was provided that the larger the touch key size, the higher the success rate and the pressing convenience.

Two groups of touch key locations, appropriate and inappropriate regions, were identified by the SNK tests. An appropriate region provided good usability in terms of each dependent measure, while an inappropriate region provided poor usability. With respect to all three measures, there was significant difference between the two groups at the 0.05 significance level. Figure 3 illustrates two groups, in which the darkest areas and white areas represent appropriate and inappropriate regions, respectively.

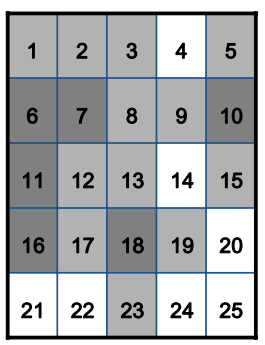

Success Rate

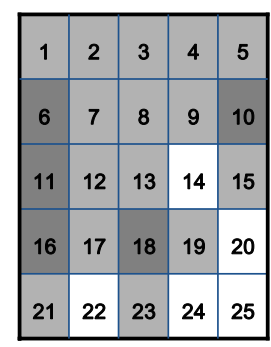

Number of Errors

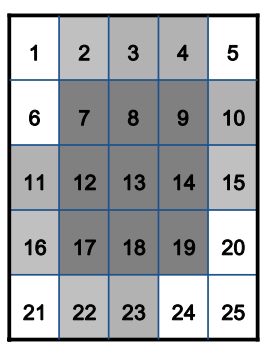

Pressing Convenience
Figure 3. Appropriate and inappropriate regions for three measures. The darkest area and white areas represent appropriate and inappropriate regions, respectively. Each number is an ID of each area.

The ANOVA on the deviation data showed the touch key size $(\mathrm{F}(2,58)=5.95, \mathrm{p}=0.004)$ and the touch key location $(\mathrm{F}(24,696)=7.61, \quad \mathrm{p}<0.001) \quad$ significantly affected on $\mathrm{x}$-axis deviation at the 0.05 significance level. Similarly, y-axis deviation was significantly influenced by the touch key size $(\mathrm{F}(2,58)=5.95, \quad \mathrm{p}=0.005)$ and the touch key location $(\mathrm{F}(24,696)=7.61, \mathrm{p}<0.001)$. Table 2 shows touch key locations with the largest deviation.

Table 2. Touch key locations with the largest deviation

\begin{tabular}{|c|c|c|}
\hline & x-axis & y-axis \\
\hline Positive direction & $1,11,21$ & $1,4,5$ \\
\hline
\end{tabular}

Negative direction

$15,20,25$

$21,22,24$

\section{Discussions}

Small touch keys have poor performance in terms of the success rate and the number of errors according to the results. Touch keys with the size of $4 \mathrm{~mm}$ provided the lowest performances in boundary region (i.e. areas with IDs of 4, 20, 21, 22, 24, and 25). For example, the success rate and the number of errors in the area with an ID of 4 were $54.3 \%$ and 4.8 , respectively. Also, touch key location with an area ID of 22 provided $61.0 \%$ and 5.6.

The poor performance could be explained by the amount of feedback information during pressing a target and the input recognition algorithm of the touch screen. Feedback information is necessary during a rapid human movement to check if a movement reaches a target [5]. The users can get only visual information from a touch screen because it is difficult to implement tactile feedback on a touch screen. Worse yet, when using a mobile device by one hand, it is difficult to get even visual information if targets are visually interfered by the hand and fingers. A traditional keyboard is one of input devices that have quite high performance. It provides tactile feedback so that users can easily recognize which keys they are pressing and whether the keys are successfully pressed or not.

Touch keys are activated only if the centroid of a pressed area falls in the recognition area of a target key, which requires users to take locations of the centroid during pressing tasks. In case of pressing a target by a thumb, however, it is difficult to calculate and take the centroid of a pressed area in mind. Worse yet, due to the anatomy of the hand, users need considerable thumb flexion and extension to press a target in some areas (e.g. areas with IDs of 20,22 , and 25) of the boundary region, which can make pressing accuracy quite low.

Adjusting a location of a touch recognition area is one of the solutions to improve the poor pressing performance. The solution seems to acceptable since it is simple and easy to manipulate design parameters by a software manner. In addition, from the collected deviation data, it is easy to obtain hits distributions from which a movement range of a recognition area could be obtained.

The solution was applied to two touch key designs $(4 \mathrm{~mm}$ touch keys located in the areas with IDs of 4 and 22), as a case study. Figure 4 illustrates hits distributions of the two designs. It showed pressing pattern of each touch key design. For example, the Yaxis deviation of the touch key location of 22 showed that subjects tended to press positions upper than center of targets because most hits had negative $\mathrm{Y}$-axis deviation. Movement ranges of a recognition area in both directions were determined using the hits distributions. Then, success rates were re-calculated within the movement ranges. The Friedman test revealed significant differences between the original success rates and the re-calculated maximum success rates for both touch key designs (for both, $\mathrm{p}=0.01$ ) at the 0.05 significance level. Specifically, the touch key with an area ID of 4 had the maximum success rate of $65.7 \%$ (21\% increase compared to the original success rate of $54.3 \%$ ) when a recognition region moved 5 pixels in the $x$ direction and -2 pixels in the y direction. The success rate of the other design increased by $9.8 \%$ (from $61.0 \%$ to $67.0 \%$ ) in case that a recognition region moved -2 pixels and -3 pixels in the $x$ and the $y$ directions, respectively. Note that, the success rates 
increased significantly, although the recognition areas moved less than 6 pixels, about $1.4 \mathrm{~mm}$ long.
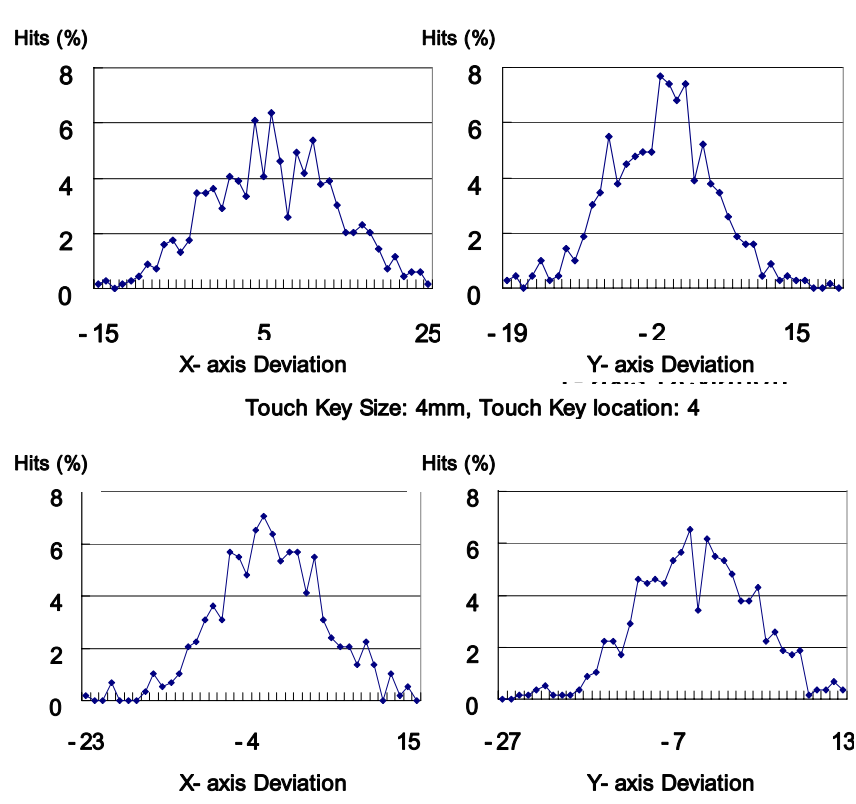

Touch Key Size: 4mm, Touch Key location: 22

Figure 4. Hits distributions of two touch key designs

The subjective satisfaction score (the pressing convenience) seems to be higher in the center region (i.e. areas with IDs of 7, 8, $9,12,13,14,17$, and 18) than other regions (See Figure 3). This result is consistent with [6]. This study, however, provides more detailed results because the previous study divided a touch screen into nine regions.

Input elements that require a highly accurate control, e.g. a shutter button for a camera function, could be located in the leftmost areas with IDs of 6,11 , and 16 because the areas had higher success rate and lower number of errors than other regions (See Figure 3). Also, center areas with IDs of 7, 8, 9, 12, 13, 17 and 18 are recommended for general input elements because they provide high pressing convenience and their performance in terms of the success rate and the number of errors is not poor.

\section{Conclusion}

This study was conducted to investigate effects of touch key sizes and locations on mobile phone usability in terms of the success rate, the number of errors, and the pressing convenience. Also, a hits distribution based algorithm was used to improve one-handed thumb touch input on a mobile phone.

The results provided the larger touch key size, the higher performance and subjective satisfaction. In addition, two types of touch key locations (an appropriate and an inappropriate region) were statistically identified with respect to the success rate, the number of errors, and the pressing convenience. Finally, an algorithm to adjust a location of a recognition area was applied to two touch key designs that provided poor pressing performance. The algorithm statistically increased the success rates. The results of this study could be used to design touch keys so as to enhance the usability of mobile phones with a touch screen.

Pressing serial targets are one of common tasks that frequently happen in mobile phone use, while this study focused on pressing a single target. Further studies are required regarding serial target selections on a mobile touch screen. Different hits distribution based algorithms to help pressing tasks (e.g. on-line adaptation, adjusting size of a recognition area, etc.), also, are worth to be examined in further studies.

\section{ACKNOWLEDGMENTS}

This work was supported by the Korea Science and Engineering Foundation (KOSEF) grant funded by the Korea government (MOEST) (No. R01-2006-000-11142-0)

\section{REFERENCES}

[1] Colle, H. A. and Hiszem, K. J. 2004. Standing at a kiosk: Effects of key size and spacing on touch screen numeric keypad performance and user preference. Ergonomics. 47, 13, 1406-1423.

[2] Hesel, D. R. and Hirsch, R. M. 2002. Statistical methods in water resources. U.S. Department of the Interior. DOI= http://pubs.usgs.gov/twri/twri4a3/

[3] Himberg, J., Häkkilä, J., Kangas, P., and Mäntyjärvi, N. 2003. On-line personalization of a touch screen based keyboard. In Proceedings of the Intelligent User Interfaces Conference (Miami, Florida, January 12 - 15, 2003). IUI'03. ACM Press, New York, NY, 77-84.

[4] Karlson, A. K., Bederson, B. B., and Contreras-Vidal, J. L. 2006. Understanding single-handed mobile device interaction. Technical Report. University of Maryland at College Park.

[5] Meyer, D. E., Abrams, R. A., and Wright, C. E. 1990. Speedaccuracy tradeoffs in aimed movements: toward a theory of rapid voluntary action. M. Jeannerod, Ed. Lawrence Erlbaum Associates, Mahwah, New Jersey, 173-226.

[6] Parhi, P., Karlson, A. K. and Benderson, B. B. 2006. Target size study for one-handed thumb use on small touchscreen devices. In Proceedings of the Mobile HCI Conference (Helsinki, Finland, September 12 - 15, 2006). MobileHCI'06. ACM Press, New York, NY, 203-210.

[7] Zhai, S., Sue, A., and Accot, J. 2002. Movement model, Hits distribution and learning in virtual keyboarding. In proceedings of CHI (Minneapolis, Minnesota, April 20 - 25, 2002). CHI'02. ACM Press, New York, NY, 17-24. 